\title{
State estimation of uncertain multiple model with unknown inputs
}

\author{
Abdelkader Akhenak, Mohammed Chadli, Didier Maquin and José Ragot \\ Centre de Recherche en Automatique de Nancy, CNRS UMR 7039 \\ Institut National Polytechnique de Lorraine \\ 2, Avenue de la forêt de Haye \\ 54516 Vandœuvre-les-Nancy Cedex, FRANCE \\ \{aakhenak, mchadli, dmaquin, jragot\}@ensem.inpl-nancy.fr
}

\begin{abstract}
This paper is dedicated to the synthesis of a sliding mode multiple observer. The considered systems are represented by an uncertain (nonlinear) multiple model with unknown inputs. Stability conditions of such observers are expressed in terms of linear matrix inequalities (LMI). An example of simulation is given to illustrate the proposed method.
\end{abstract}

\section{INTRODUCTION}

A physical process is often subjected to disturbances which have as origin the noises due to its environment, uncertainty of measurements, fault of sensors and/or actuators. These disturbances have harmful effects on the normal behavior of the process and their estimation can be used to conceive a control strategy able to minimize their effects. The disturbances are called unknown inputs when they affect the input of the process and their presence can make difficult the state estimation.

In the linear system framework, observers can be designed for singular systems, unknown input systems, delay systems and also uncertain system with time-delay perturbations [18][7]. Several works were also achieved concerning the estimation of the state and the output in the presence of unknown inputs. They can be gathered into two categories. The first one supposes an a priori knowledge of information on these nonmeasurable inputs; in particular, Johnson [10] proposes a polynomial approach and Meditch [14] suggests approximating the unknown inputs by the response of a known dynamic system. The second category proceeds either by estimation of the unknown inputs, or by their complete elimination from the equations of the system.

Among the techniques that do not require the elimination of the unknown inputs, Wang [16] proposes an observer able to entirely reconstruct the state of a linear system in the presence of unknown inputs and in [4][11][13], to estimate the state, a model inversion method is used. Using the Walcott and Zak structure observer [17] Edwards et al. [5][6] have also designed a convergent observer using the Lyapunov approach. Other techniques are based on the elimination of the unknown inputs [9][12].
However, the real physical systems are often nonlinear. As it is delicate to synthesize an observer for a nonlinear system, we preferred to represent these systems with a multiple model. The idea of the multiple model approach is to apprehend the total behavior of a system by a set of local models (linear or affine), each local model characterizing the behavior of the system in a particular zone of operation. The local models are then aggregated by means of an interpolation mechanism.

In the case of a nonlinear system affected by unknown inputs and described by a multiple model, a technique for multiple model state estimation by using a multiple observer with sliding mode has already been proposed [1][3].

In this paper, we consider the state estimation of an uncertain multiple model with unknown input. For that purpose a multiple observer based on convex interpolation of classical Luenberger observers [1] involving additive terms used to overcome the uncertainties is designed. Using quadratic Lyapunov function, sufficient asymptotic stability conditions are given in LMI formulation [2].

Notation: Throughout the paper, the following useful notation is used: $X^{T}$ denotes the transpose of the matrix $X$, $X>0$ means that $X$ is a symmetric positive definite matrix, $\mathbb{I}_{M}=\{1,2, \ldots, M\}$ and $\|$.$\| represents the Euclidean norm$ for vectors and the spectral norm for matrices.

\section{Multiple MODEL APPROACH}

A multiple model is obtained by interpolating several local linear models.

$$
\left\{\begin{array}{l}
\dot{x}(t)=\sum_{i=1}^{M} \mu_{i}(\xi(t))\left(A_{i} x(t)+B_{i} u(t)+d_{i}\right) \\
y(t)=C x(t)
\end{array}\right.
$$

where $x(t) \in \mathbb{R}^{n}$ is a state vector, $u(t) \in \mathbb{R}^{m}$ in the input vector, $y(t) \in \mathbb{R}^{p}$ is the output vector and the matrix $C \in \mathbb{R}^{p \times n}$ is the output matrix of the system. For the $i^{t h}$ local model $A_{i} \in \mathbb{R}^{n \times n}$ is the state matrix, $B_{i} \in \mathbb{R}^{n \times m}$ is 
the matrix of input, and $d_{i} \in \Re^{n \times 1}$ is a constant matrix.

The activation functions $\mu_{i}(\xi(t)), i \in \mathbb{I}_{M}$ have the following properties:

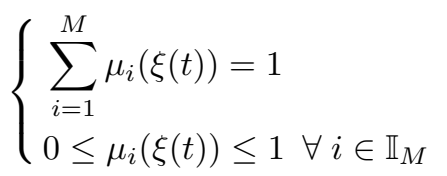

where $\xi(t)$ represents the vector of decision depending on the input and/or the measurable state variables. The number of local models $(M)$ depends on the precision of desired modeling, the complexity of the nonlinear system and the choice of the structure of the activation functions.

A multiple model can be obtained by identification [8], by linearization around various operating points (in this case each local model is an affine LTI system due to the presence of the constant of linearization) or by convex polytopic transformation [15].

In this paper, we consider that the output of the system is linear with regard to the state, i.e., $y(t)=C x(t)$ that covers a large class of real systems.

To hold account of modelisation/approximation errors and sometimes the presence of unknown inputs, a multiple model can involved uncertainties and unknown inputs:

$$
\left\{\begin{aligned}
\dot{x}(t) & =\sum_{i=1}^{M} \mu_{i}(\xi)\left(\left(A_{i}+\Delta A_{i}(t)\right) x(t)+B_{i} u(t)+\right. \\
y(t) & =C x(t)
\end{aligned}\right.
$$

The unknown inputs $\bar{u}(t)$ are assumed to be bounded, such that $\|\bar{u}(t)\|<\rho$, where $\rho$ is a positive scalar. The variable matrices $\Delta A_{i}(t)$ are also bounded, i.e., $\left\|\Delta A_{i}(t)\right\|<\delta_{i}$. The transmission matrices of the unknown inputs are $R_{i} \in$ $\mathbb{R}^{n \times q}$.

Remark: In the following, to simplify the expression of equations, time variable $(t)$ will be omitted.

\section{Multiple ObSERVER STRUCTURE}

In this paper, we consider the state estimation of an uncertain multiple model perturbed by unknown inputs. The proposed multiple observer is based on a linear combination of local Luenberger observer involving sliding terms allowing to compensate the uncertainties and the unknown inputs.

\section{A. Multiple observer stability conditions}

It is assumed that the pairs $\left(A_{i}, C\right)$ are observable and there exists matrices $G_{i} \in \mathbb{R}^{n \times p}$, such that $\bar{A}_{i}=A_{i}-G_{i} C$ have stable eigenvalues, i.e., there exists symmetric and positive definite matrices $\left(P, Q_{i}\right)$ and matrices $F_{i} \in \mathbb{R}^{m \times p}$ satisfying the following structural constraints:

$$
\left\{\begin{aligned}
\bar{A}_{i}^{T} P+P \bar{A}_{i} & =-Q_{i} \\
C^{T} F_{i}^{T} & =P R_{i}, \quad \forall i \in 1, \ldots, M
\end{aligned}\right.
$$

The proposed multiple observer of the multiple model (3) has the following form:

$\left\{\begin{array}{l}\dot{\hat{x}}=\sum_{i=1}^{M} \mu_{i}(\xi)\left(A_{i} \hat{x}+B_{i} u+d_{i}+G_{i}(y-C \hat{x})+R_{i} \nu_{i}+\alpha_{i}\right) \\ \hat{y}=C \hat{x}\end{array}\right.$

The aim of the design is to determine gain matrices $G_{i}$ and variables $\nu_{i} \in \mathbb{R}^{q}$ and $\alpha_{i} \in \mathbb{R}^{n}$, that guarantee the asymptotic convergence of $\hat{x}$ towards $x$. Let us note that the variables $\nu_{i}$ and $\alpha_{i}$ compensate respectively the errors due to the unknown inputs and the model uncertainties. Let us define the state estimation error:

$$
e=x-\hat{x}
$$

The output estimation error is defined as follows:

$$
r=y-\hat{y}=C(x-\hat{x})=C e
$$

The dynamic of the state estimation error is governed by:

$\dot{e}=\sum_{i=1}^{M} \mu_{i}(\xi)\left(\left(A_{i}-G_{i} C\right) e+\Delta A_{i} x+R_{i} \bar{u}-R_{i} \nu_{i}-\alpha_{i}\right)$

Theorem 1: The state estimation of the robust state multiple observer (5) converges globally asymptotically to the state of the multiple model (3), if $\nu_{i}(t)$ and $\alpha_{i}(t)$ are given by the following equations:

$$
\left\{\begin{array}{l}
\text { If } r \neq 0 \\
\text { If } r=0 \quad\left\{\begin{array}{l}
\nu_{i}=\rho \frac{F_{i} r}{\left\|F_{i} r\right\|} \\
\alpha_{i}=\beta_{1}\left(1+\beta_{2}\right) \delta_{i}^{2} \frac{\hat{x}^{T} \hat{x}}{2 r^{T} r} P^{-1} C^{T} r \\
\alpha_{i}=0
\end{array}\right.
\end{array}\right.
$$

and if there exists a matrix $P>0$, some matrices $F_{i}$ and positive scalars $\beta_{1}$ and $\beta_{2}$ satisfying the following constraints:

$$
\left\{\begin{array}{l}
\bar{A}_{i}^{T} P+P \bar{A}_{i}+\beta_{1}^{-1} P^{2}+\beta_{1}\left(1+\beta_{2}^{-1}\right) \delta_{i}^{2} I<0 \\
C^{T} F_{i}^{T}=P R_{i}, i \in \mathbb{I}_{M}
\end{array}\right.
$$

with:

$$
\bar{A}_{i}=A_{i}-G_{i} C
$$

The demonstration of the asymptotic convergence of this multiple observer uses the following lemma 1:

Lemma 1: For any matrices $X$ and $Y$ with appropriate dimensions, the following property holds for any positive scalar $\beta$ :

$$
X^{T} Y+Y^{T} X \leq \beta X^{T} X+\beta^{-1} Y^{T} Y
$$


Proof: In order to demonstrate the asymptotic convergence of the multiple observer, let us consider the following Lyapunov function:

$$
V(e)=e^{T} P e
$$

Its time derivative, evaluated along the trajectory of the system by using equations (6) and (8), may be expressed as:

$$
\begin{array}{r}
\dot{V}=\sum_{\substack{i=1 \\
M}}^{M} \mu_{i}(\xi)\left(e^{T}\left(\bar{A}_{i}^{T} P+P \bar{A}_{i}\right) e+x^{T} \Delta A_{i}^{T} P e+\right. \\
\left.e^{T} P \Delta A_{i} x-2 \alpha_{i}^{T} P e+2 e^{T} P R_{i} \bar{u}-2 e^{T} P R_{i} \nu_{i}\right)
\end{array}
$$

The lemma (1) property, allows to write:

$$
\begin{array}{r}
\dot{V} \leq \sum_{i=1}^{M} \mu_{i}(\xi)\left(e^{T}\left(\bar{A}_{i}^{T} P+P \bar{A}_{i}\right) e+\beta_{1} x^{T} \Delta A_{i}^{T} \Delta A_{i} x+\right. \\
\left.\beta_{1}^{-1} e^{T} P^{2} e-2 \alpha_{i}^{T} P e+2 e^{T} P R_{i} \bar{u}-2 e^{T} P R_{i} \nu_{i}\right)
\end{array}
$$

Using the expression of the state estimation error (6), (14) becomes:

$$
\begin{array}{r}
\dot{V} \leq \sum_{i=1}^{M} \mu_{i}(\xi)\left(e^{T}\left(\bar{A}_{i}^{T} P+P \bar{A}_{i}+\beta_{1}^{-1} P^{2}\right) e+2 e^{T} P R_{i} \bar{u}+\right. \\
\left.\beta_{1} \delta_{i}^{2}(\hat{x}+e)^{T}(\hat{x}+e)-2 \alpha_{i}^{T} P e+2 e^{T} P R_{i} \nu_{i}\right) \\
\dot{V} \leq \sum_{i=1}^{M} \mu_{i}(\xi)\left(e^{T}\left(\bar{A}_{i}^{T} P+P \bar{A}_{i}+\beta_{1}^{-1} P^{2}\right) e+2 e^{T} P R_{i} \bar{u}+\right. \\
\beta_{1} \delta_{i}^{2}\left(\hat{x}^{T} \hat{x}+e^{T} e\right)+\beta_{1} \delta_{i}^{2}\left(\hat{x}^{T} e+e^{T} \hat{x}\right) \\
\left.-2 \alpha_{i}^{T} P e-2 e^{T} P R_{i} \nu_{i}\right)
\end{array}
$$

Using again the property of the lemma (1), the expression (15) can be rewritten as follows:

$$
\begin{aligned}
\dot{V} \leq & \sum_{i=1}^{M} \mu_{i}(\xi)\left(e^{T}\left(\bar{A}_{i}^{T} P+P \bar{A}_{i}+\beta_{1}^{-1} P^{2}+\beta_{3} \delta_{i}^{2} I\right) e+\right. \\
& \left.\beta_{1}\left(1+\beta_{2}\right) \delta_{i}^{2} \hat{x}^{T} \hat{x}-2 \alpha_{i}^{T} P e+2 e^{T} P R_{i} \bar{u}-2 e^{T} P R_{i} \nu_{i}\right)
\end{aligned}
$$

with $\beta_{3}=\beta_{1}\left(1+\beta_{2}^{-1}\right)$

When $r \neq 0$, by using the relation (9), it is easy to notice that:

$$
\begin{aligned}
2 \alpha_{i}^{T} P e & =\beta_{1}\left(1+\beta_{2}\right) \delta_{i}^{2} \frac{\hat{x}^{T} \hat{x}}{r^{T} r} r^{T} C P^{-1} P e \\
& =\beta_{1}\left(1+\beta_{2}\right) \delta_{i}^{2} \frac{\hat{x}^{T} \hat{x}}{r^{T} r} r^{T} r \\
& =\beta_{1}\left(1+\beta_{2}\right) \delta_{i}^{2} \hat{x}^{T} \hat{x}
\end{aligned}
$$

Therefore, after simplification, we obtain:

$$
\begin{array}{r}
\dot{V} \leq \sum_{i=1}^{M} \mu_{i}(\xi)\left(e^{T}\left(\bar{A}_{i}^{T} P+P \bar{A}_{i}+\beta_{1}^{-1} P^{2}+\beta_{3} \delta_{i}^{2} I\right) e+\right. \\
\left.2 e^{T} P R_{i} \bar{u}-2 e^{T} P R_{i} \nu_{i}\right)
\end{array}
$$

By using the second equality of the structural constraint, $C^{T} F_{i}^{T}=P R_{i}$, we obtain:

$$
\begin{aligned}
2 e^{T} P R_{i} \bar{u}-2 e^{T} P R_{i} \nu_{i} & =2 e^{T} C^{T} F_{i}^{T} \bar{u}-2 e^{T} C^{T} F_{i}^{T} \nu_{i} \\
& =2 r^{T} F_{i}^{T} \bar{u}-2 r^{T} F_{i}^{T} \nu_{i}
\end{aligned}
$$

By replacing the variables $\nu_{i}$ by the expression (9), this last expression can be written as:

$$
\begin{aligned}
2 e^{T} P R_{i} \bar{u}-2 e^{T} P R_{i} \nu_{i} & =2 r^{T} F_{i}^{T} \bar{u}-2 r^{T} F_{i}^{T} \nu_{i} \\
& =2 r^{T} F_{i}^{T} \bar{u}-2 \rho r^{T} F_{i}^{T} \frac{F_{i} r}{\left\|F_{i} r\right\|} \\
& =2 r^{T} F_{i}^{T} \bar{u}-2 \rho\left\|F_{i} r\right\|
\end{aligned}
$$

This last expression is then clearly negative, therefore, the inequality (17) becomes:

$$
\begin{array}{r}
\dot{V} \leq \sum_{i=1}^{M} \mu_{i}(\xi)\left(e^{T}\left(\bar{A}_{i}^{T} P+P \bar{A}_{i}+\beta_{1}^{-1} P^{2}+\beta_{3} \delta_{i}^{2} I\right) e+\right. \\
\left.2 r^{T} F_{i}^{T} \bar{u}-2 \rho\left\|F_{i} r\right\|\right) \\
\leq \sum_{i=1}^{M} \mu_{i}(\xi)\left(e^{T}\left(\bar{A}_{i}^{T} P+P \bar{A}_{i}+\beta_{1}^{-1} P^{2}+\beta_{3} \delta_{i}^{2} I\right) e\right)
\end{array}
$$

When $r=0$, we obtain the same result.

As the RHS of inequality (18) is clearly negative, the asymptotic convergence of the multiple observer is guaranteed. Then the state estimation error converges asymptotically towards zero, if the conditions (9) and the inequalities (10) are checked. The inequalities (10) are nonlinear in $P$ and $G_{i}$. LMI Techniques can thus be used only after linearization of these inequalities. We chose a technique of change of variable.

\section{B. Resolution method}

Consider the following change of variable:

$$
W_{i}=P G_{i}
$$

Then, the inequalities (10) can be written as:

$$
A_{i}^{T} P+P A_{i}-C^{T} W_{i}^{T}-W_{i} C+\beta_{1}^{-1} P^{2}+\beta_{3} \delta_{i}^{2} I<0
$$

Applying the Schur complement [12], we obtain from (19) the following LMI formulation:

$$
\left[\begin{array}{cc}
A_{i}^{T} P+P A_{i}-C^{T} W_{i}^{T}-W_{i} C+\beta_{3} \delta_{i}^{2} I & P \\
P & -\beta_{1} I
\end{array}\right]<0
$$

The solution of this LMI in $P$ and $G_{i}$ allows one to compute the observer gains $G_{i}=P^{-1} W_{i}$. 


\section{Simulation ExAmPLe}

Consider the multiple model, made up of two local models and involving two outputs and three states.

$$
\left\{\begin{array}{l}
\dot{x}=\sum_{i=1}^{2} \mu_{i}(\xi)\left(\left(A_{i}+\Delta A_{i}\right) x+B_{i} u+R_{i} \bar{u}\right) \\
y=C x
\end{array}\right.
$$

The numerical values of matrices $A_{i}, B_{i}, C_{i}$ and $R_{i}$ are as follows:

$$
\begin{gathered}
A_{1}=\left[\begin{array}{ccc}
-2 & 1 & 1 \\
1 & -3 & 0 \\
2 & 1 & -6
\end{array}\right] \quad A_{2}=\left[\begin{array}{ccc}
-3 & 2 & 2 \\
5 & -8 & 0 \\
0.5 & 0.5 & -4
\end{array}\right] \\
B_{1}=\left[\begin{array}{c}
1 \\
0.5 \\
0.5
\end{array}\right] \quad B_{2}=\left[\begin{array}{c}
0.5 \\
1 \\
0.25
\end{array}\right] \\
R_{1}=\left[\begin{array}{l}
1 \\
1 \\
1
\end{array}\right] \quad R_{2}=\left[\begin{array}{c}
1 \\
0.5 \\
2
\end{array}\right] \quad C=\left[\begin{array}{lll}
1 & 1 & 1 \\
1 & 0 & 1
\end{array}\right]
\end{gathered}
$$

Model of uncertainties are such that $\Delta A_{i}(j, k)(t)=0.1 A_{i}(j, k) \eta(t)$ $(j, k) \in\{1,3\}$, the function $\eta(t)$ is a Gaussian random function with zero mean and a unity variance.

In this case, the multiple observer that estimates the state vector of the multiple model is described by,

$\left\{\begin{array}{l}\dot{\hat{x}}=\sum_{i=1}^{2} \mu_{i}(\xi)\left(A_{i} \hat{x}+B_{i} u+G_{i}(y-C \hat{x})+R_{i} \nu_{i}+\alpha_{i}\right) \\ \hat{y}=C \hat{x}\end{array}\right.$

with

$$
\left\{\begin{array}{r}
\left(A_{i}-G_{i} C\right)^{T} P+P\left(A_{i}-G_{i} C\right)+\beta_{1}^{-1} P^{2}+\beta_{3} \delta_{i}^{2} I<0 \\
C^{T} F_{i}^{T}=P R_{i}, \quad \forall i \in\{1,2\}
\end{array}\right.
$$

It is important to note that a potential problem arises in the implementation of this multiple observer: when the output estimation error $r(t)$ tends towards zero, the magnitude of $\alpha_{i}(t)$ and $\nu_{i}(t)$ may increase without bound. This problem is overcome as follows.

The terms $\nu_{i}(t)$ and $\alpha_{i}(t)$ are fixed to zero when the output estimation error us such that $\|r(t)\|<\varepsilon$ a small positive number chosen by the user. In this case, the estimation error cannot converge to zero asymptotically but to a small neighborhood of zero depending on the choice of $\varepsilon$.

\section{A. Simulation results}

The simultaneous resolution of equations (24) using LMI tools leads to the following matrices $G_{i}, F_{i}$ and $P$ :

$$
\mathrm{G}_{1}=\left[\begin{array}{cc}
0.24 & 0.73 \\
15.32 & -13.06 \\
4.46 & 1.24
\end{array}\right], \mathrm{G}_{2}=\left[\begin{array}{cc}
1.18 & 6.68 \\
14.52 & -7.29 \\
11.49 & -3.88
\end{array}\right]
$$

$$
\begin{gathered}
\mathrm{P}=\left[\begin{array}{ccc}
1.83 & -0.81 & 0.51 \\
-0.81 & 3.35 & 0.06 \\
0.51 & 0.06 & 0.95
\end{array}\right] \\
\mathrm{F}_{1}=\left[\begin{array}{ll}
2.60 & -1.06
\end{array}\right] \text { and } \mathrm{F}_{2}=\left[\begin{array}{ll}
0.98 & 1.47
\end{array}\right]
\end{gathered}
$$

The system (22) was simulated using the known and unknown inputs depicted in figures (1) and (2).

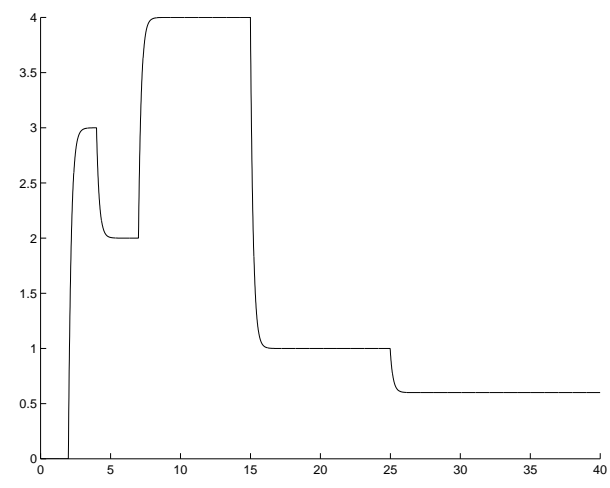

FIG. 1 -. the known input $u(t)$

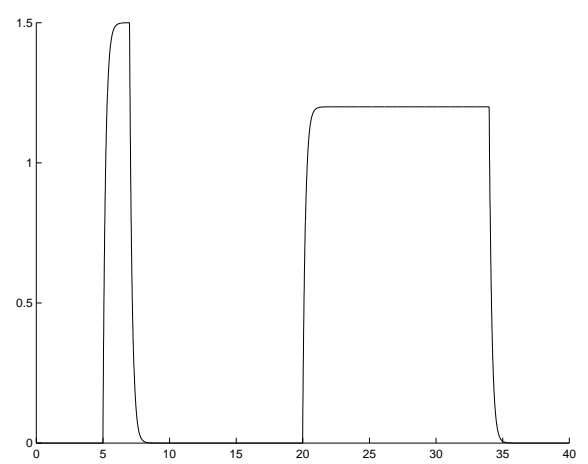

FIG. 2 -. the unknown input $\bar{u}(t)$

The figures (3), (4) and (5) show the comparison between the state of the multiple model and its estimate from the multiple observer. The figures (6) and (7) show the two outputs of the multiple model and their respective estimates. It is noted that the two layouts are superimposed except in the vicinity of the origin; that is due to the choice of the initial conditions of the multiple observer.

\section{CONCLUSION}

Based on an uncertain multiple model representation, the design of a multiple observer using the principle of interpolation of local observers has been proposed. Moreover, the case where some inputs of the system are unknown has been considered. The calculation of the gains of the multiple observer is then returned to a simultaneous calculation of the gains of the local observers. The stability of the global 


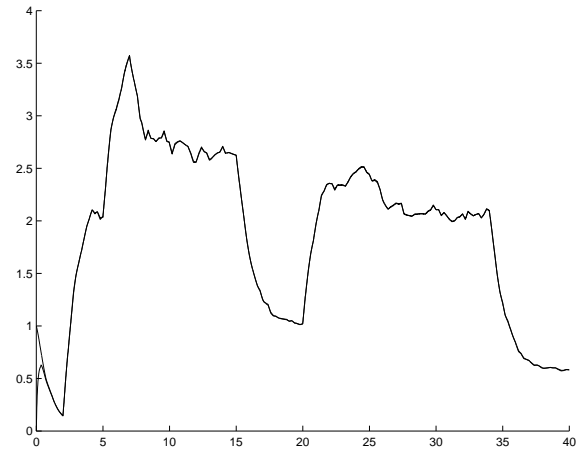

FIG. 3 -. $x_{1}(t)$ of the multiple model and its estimate

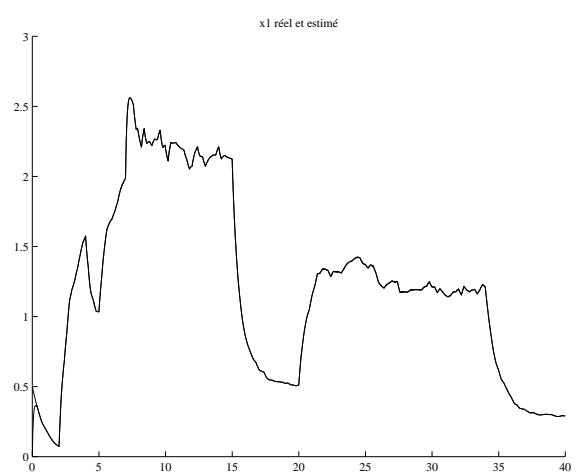

FIG. 4 -. $\quad x_{2}(t)$ of the multiple model and its estimate

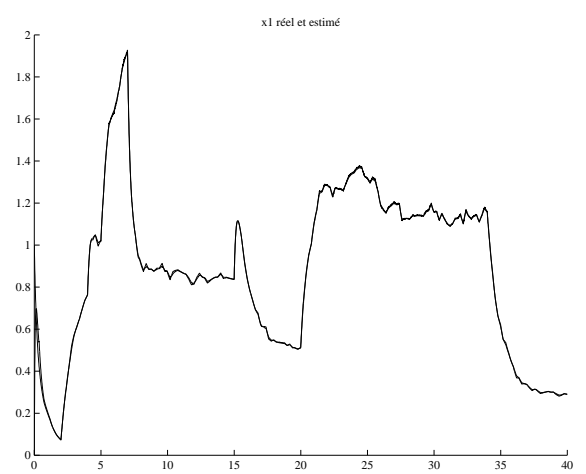

FIG. 5 -. $x_{3}(t)$ of the multiple model and its estimate

observer requires however the consideration of coupling constraints between these local observers; these contraints lead to the resolution of a LMI problem under structural constraints. Assuming the existence of suited matrices, we showed that the reconstruction of the state and unknown inputs vectors of the multiple model is possible. The simulation results show that the estimation of state and unknown inputs is very satisfactory.

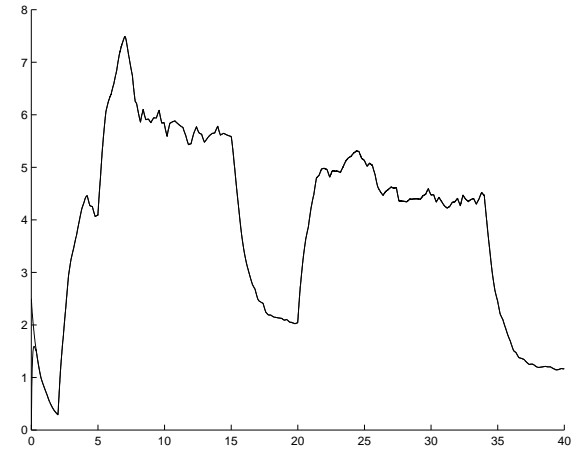

FIG. 6 -. $\quad y_{1}(t)$ of the multiple model and its estimate

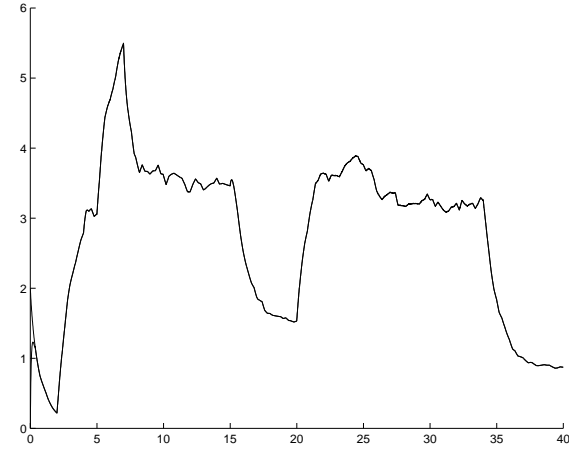

FIG. 7 -. $\quad y_{2}(t)$ of the multiple model and its estimate

\section{REFERENCES}

[1] A. Akhenak, M. Chadli, D. Maquin and J. Ragot, Sliding mode multiple observer for fault detection and isolation, 42th IEEE Conference on Decision and Control, Hawaii, December 9-12, 2003.

[2] S. Boyd, L. El Ghaoui, E. Feron and V. Balakrishnan, Linear Matrix Inequalities in System and Control Theory, Philadelphia: SIAM, 1994.

[3] M. Chadli, D. Maquin, J. Ragot, Multiple observer for discrete-time multiple model, IFAC Congress, Safeprocess, Washington, June 2003.

[4] J. Chen and H. Zhang, Robust detection of faulty actuators via unknown input observers Int. J. Systems Sci, vol. 22, n. 10, MIT, October, 1980.

[5] C. Edwards and S. K. Spurgeon. On the development of discountinuous observers, Int. J. Control, Vol. $59 \mathrm{~N}^{\circ}$ 5, pp. 1211-1229, 1994.

[6] C. Edwards and S. K. Spurgeon. Sliding mode observers for fault detection and isolation, Automatica, Vol. 36, $\mathrm{N}^{\circ}$ 6, pp. 541-553, 2000.

[7] K. K. Fan, J. G. Hsieh, LMI Approach to design of robust state observer for uncertain systems with time-delay perturbation, IEEE ICIT'02, Bangkok, Thailand, pp. 1111-1115, 2002.

[8] K. Gasso, G. Mourot and J. Ragot, Structure identification in multiple model representation: elimination and merging of local models, IEEE Conference on Decision and Control, CDC'2001, Orlando, USA, December 2001.

[9] Y. Guan, and M. Saif, A novel approach to the design of unknown input observers, IEEE Trans on Automatic Control, AC-36, n. 5, pp. 632-635, 1991.

[10] C. D. Johnson, Observers for linear systems sith unknown and inaccessible inputs, Int. J. Control, vol. 21, pp. 825-831, 1975.

[11] N. Kobayashi and T. Nakamizo, An observer design for linear systems with unknown inputs, Int. J. Control, vol. 35, pp. 605-619, 1982. 
[12] P. Kudva, N. Viswanadham and A. Ramakrishna, Observers for linear systems with unknown inputs, IEEE Trans on Automatic Control AC25, pp. 113-115, 1980.

[13] L. M. Lyubchik and Y. T. Kostenko, The output control of multivariable systems with unmeasurable arbitrary disturbances - The inverse model approach, ECC'93, pp. 1160-1165, june 28-july 1, Groningen, The Netherlands, 1993.

[14] J. S. Meditch and G. H. Hostetter, Observers for systems with unkonwn and inaccessible inputs, Int. J. Control, vol. 19, pp. 637640, 1971.

[15] K. Tanaka, T. Ikeda, O. Wang, Fuzzy regulator and fuzzy observer: relaxed stability conditions and LMI based design, IEEE Trans on Fuzzy Systems, Vol. 6, N 2, pp. 250-256, 1998.

[16] V. I. Utkin. Principles of identification using sliding regimes. Soviet Physic: Doklady, Sliding mode in control optimisation, (Berlin: Springer-Verlag) Vol. 26, 271-272, 1992.

[17] B. L. Walcott and S. H. Zak. Observation of dynamical systems in the presence of bounded nonlinearities/uncertainties, 25th IEEE Conference on Decision and Control, pp. 961-966, 1988.

[18] S. H. Wang, E. J. Davison and P. Dorato, Observing the states of systems with unmeasurable disturbances, IEEE Trans on Automatic Control AC-20, pp.716-717, 1975. 